\title{
Positron Annihilation Lifetime Spectroscopy of ABS Objects Manufactured by Fused Deposition Modelling
}

\author{
M. DRYZEK ${ }^{a}$ AND E. DRYZEK ${ }^{b, *}$ \\ ${ }^{a}$ Institute for Computational Civil Engineering, Cracow University of Technology, Kraków, Poland \\ ${ }^{b}$ Institute of Nuclear Physics, Polish Academy of Sciences, PL-31342 Kraków, Poland
}

\begin{abstract}
Positron annihilation lifetime spectroscopy was used to study acrylonitrile butadiene styrene (ABS) specimens manufactured using fused deposition modelling to explore possibilities of identifying differences in molecular structure. The set of specimens was prepared including square tiles and long rectangular tiles $(100 \mathrm{~mm}$ and $200 \mathrm{~mm}$ long) with all filament roads parallel to the longer edge. All types of tiles were produced with various infill line distance parameter resulting in different overlapping of the roads in horizontal and vertical directions. The slight increase of the ortho-positronium lifetime indicating increase of the mean free volume radius was observed for the longest tiles for which influence of weld interface is expected to be most pronounced. No differences were observed for different infill line distance parameters.
\end{abstract}

DOI: 10.12693/APhysPolA.132.1506

PACS/topics: $78.70 . \mathrm{Bj}, 61.41 .+\mathrm{e}$

\section{Introduction}

Additive manufacturing (AM) commonly referred to as $3 \mathrm{D}$ printing is a manufacturing process of producing parts by adding-layer-upon-layer of material. Traditionally, it has been used for singular rapid prototyping purposes including visualisation, design verification, and functionality testing. However, objects manufactured by AM are more frequently considered as potential end-use products. In recent years $3 \mathrm{D}$ printing has become more and more popular mostly because of the equipment price drop [1].

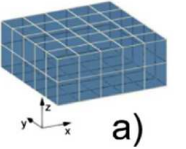

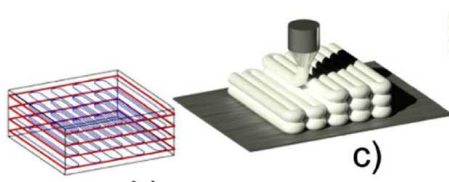

b)

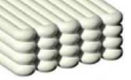

d)
Fig. 1. Schematics of fused deposition modelling process: (a) CAD model, (b) slicing, (c) 3D printing, (d) post-processing.

Fused deposition modelling (FDM) is one of the most popular AM technologies. The process can be divided into four steps (see Fig. 1). First the 3D CAD model of a desired element is being prepared using a computer software. Then the software slices the model into layers, calculates the path of extruded material, called toolpath or raster, and adds model of support material if necessary.

*corresponding author; e-mail: ewa.dryzek@ifj.edu.pl
The path pattern usually is prepared in the way that material creates the solid outer surface called contour and lattice inner fill called infill. In the next step, the software convert the path to a code and sends commands to an automated machine called a 3D printer and the process of production begins. The main part of the 3D printer is the extruder. It can be precisely manoeuvred in $X-Y-Z$ print area by stepper motors and it can heat, melt, and deposit a thermoplastic filament through its nozzle in the wanted location. The extruder follows the toolpath prepared by the software and deposits roads of material. Due to the thermal fusion the road bonds with the layer beneath and roads beside and solidifies creating a solid fill, which then forms the chosen shape. Finally, the created part is being post-processed by removing any additional supports and smoothening the surface. The technology is simple to use. The products can have complex geometries and its contour and infill path can be varied depending on expected properties. However, the material is heterogeneous, filled with layers of fine roads, welds between and voids which creates anisotropy of the material properties. Also the outer surface is not smooth because of layered structure (stair-stepping).

Due to rapid invention in the field of 3D printing the technology found application in variety of disciplines from medicine [2], car industry [3], to even building industry [4]. Ease of using the tools, no need of producing expensive moulds and control over inner structure of the final product give AM advantage over conventional manufacturing methods especially in projects which require only one or a few copies of the same part. In many of these cases the produced parts are expected to have certain structural, mechanical or thermal properties. This creates a new subject for exploring by science testing and analysis.

Typical material used in small scale FDM process are thermoplastic polymers in a form of filament including 
polycarbonate and ABS. ABS is common thermoplastic characterised by its toughness and strength. Its properties are determined by composition of three monomers: acrylonitrile, butadiene, and styrene. Acrylonitrile and styrene polymers are responsible for strength and rigidity while butadiene rubbery phase for toughness. The average size of the butadiene particles is $200 \mathrm{~nm}$, however, the size distribution is so large that particles $50 \mu \mathrm{m}$ in diameter can be also found [5].

During manufacturing process the material must be heated to its melting point to form it through the nozzle and create sufficient bond with previously extruded layer. ABS is amorphous and therefore has no true melting point. The temperature in FDM process is kept above its glass transition temperature, which is $c a .105^{\circ} \mathrm{C}$. Usually higher temperature $\left(\approx 230^{\circ} \mathrm{C}\right)$ is required to additionally heat already built material to create a form of weld with newly extruded bead. Exceeding this temperature may, however, spoil the material by breaking polymer chains [1].

It was reported that the weld interface tends to have other molecular structure then the road build and it varies between raster configuration in the same specimen shape [6]. The authors suggest that differences may result from variation of time of bonding of the neighbouring roads and because ABS is sensitive to thermal history. This observation casts a new light on a complex issue of mechanical properties of 3D printed objects. In the present investigation, we attempt to find out if the positron annihilation lifetime spectroscopy (PALS) can detect changes in the free volume microstructure connected to the presence of the weld interface in the 3D printed samples.

\section{Experimental}

Specimens were produced in FDM 3D printer 3D Kreator Motion. Material used in the process was ABSFX in filament form manufactured by F3D Filament. The filament was heated to $230^{\circ} \mathrm{C}$. The speed of depositing was set to $60 \mathrm{~mm} / \mathrm{s}$. The nozzle diameter was equal to $0.4 \mathrm{~mm}$. The specimens were chosen to be in the form of $5 \mathrm{~mm}$ thick tiles $10 \mathrm{~mm}$ in width. There were three length variants: $10 \mathrm{~mm}, 100 \mathrm{~mm}, 200 \mathrm{~mm}$. The slicing software to create input file for the printer was Cura 2.5. The raster pattern was chosen to be lines without change in angle between the layers which resulted in unidirectional roads packing (see Fig. 2). Thus, raster orientation defined as the angle between the print direction and the long axis of the specimens was equal to $0^{\circ}$. The layer height was equal to $0.35 \mathrm{~mm}$. Different infill line distance (see Fig. 3), i.e., 0.34, 0.37, and $0.4 \mathrm{~mm}$ was chosen to differentiate the weld interface range in the build. Infill line distance minus diameter is sometimes referred to as raster to raster air gap. If this value is negative roads are overlapping and this print configuration is reported to improve mechanical properties of an component as a whole [7]. a)

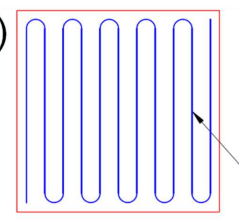

b)

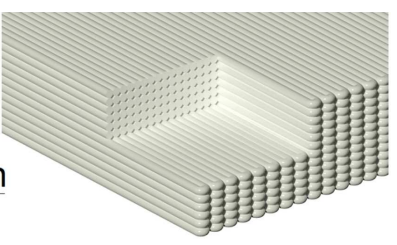

Fig. 2. (a) A toolpath example in one layer. (b) Visualisation with the cross-section trough.

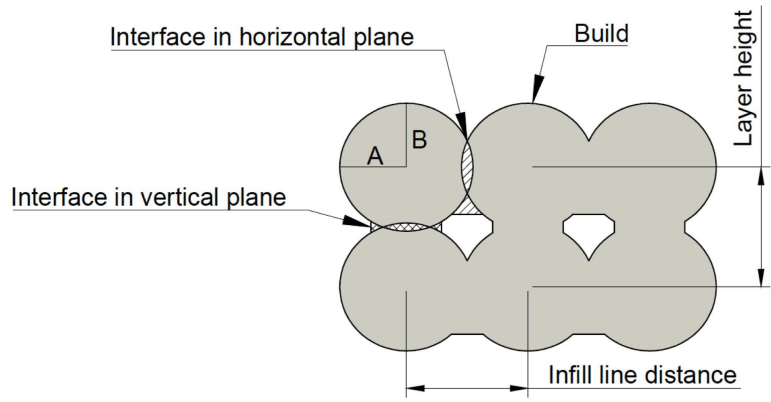

Fig. 3. A simplified cross-section of the unidirectional print proposed in [8].

For PALS measurements, a lifetime spectrometer with a time resolution (FWHM) of 280 ps was used. A positron source of ${ }^{22} \mathrm{Na}$ deposited on Kapton foil $(7 \mathrm{~mm}$ thick) was sandwiched between two identical samples. The obtained PALS spectra with a total number of $1.5 \times 10^{6}$ counts were analysed using the LT code [9], taking into account three annihilation channels: i.e. $p$ Ps, free positrons, and o-Ps annihilation; as well as background subtraction and the source contribution allowed us to obtain $\chi^{2}$ of the fit close to unity.

\section{Results and discussion}

The way the specimens were prepared, i.e., with the toolpath pattern being lines along the tile length without changing the build orientation between the layers was chosen to enhance the influence of the interface between builds. The weld interface between the roads was found to be depleted in the rubbery butadiene phase in comparison to the build surfaces [6]. It also exhibited an interfacial stiffening effect connected to increase in the indentation elastic modulus. This effect was more pronounced when the rasters were relatively long and it was explained by the shorter time in which the beads interacted with each other being still in the melt state during deposition [6]. Thus the samples prepared by 3D printing are not entirely homogeneous and this can affect positron annihilation including the size of local free volumes detected by $o$-Ps.

Considering three components, the analysis of the positron lifetime spectra gave the following values: $\tau_{1}$ close to $0.19 \mathrm{~ns}$ with intensity $I_{1}$ equal to $c a .17 \%, \tau_{2}$ close to 0.45 ns with intensity $I_{2}$ equal to $c a .60 \%$ which 


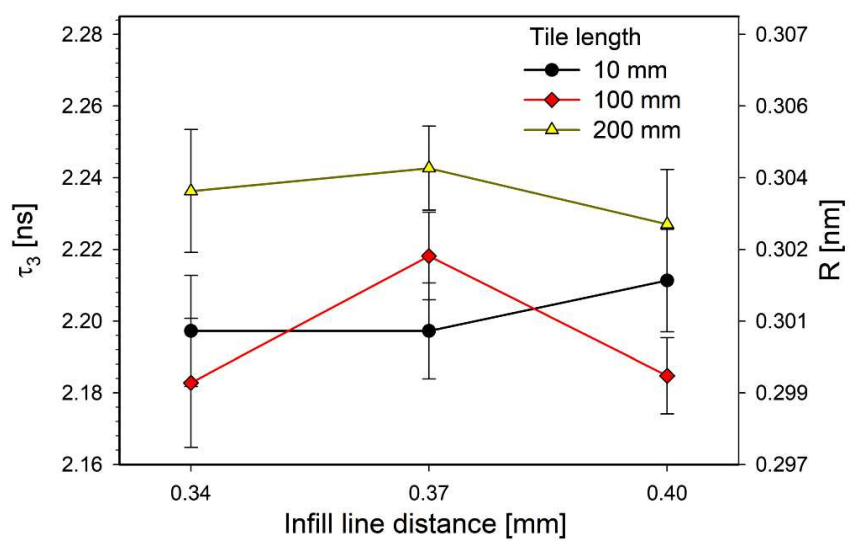

Fig. 4. The $o$-Ps lifetime as a function of the infill distance for specimens of different length.

are similar for all the samples. The values of the longest lifetime component, $\tau_{3}$, as a function of the infill line distance for the 3D printed samples of different length are shown in Fig. 4. Assuming that $o$-Ps annihilates in the pick-off process, the right axis shows the average local free volume radius calculated according to the semiempirical model $[10,11]$ from the formula

$$
\tau_{3}=\frac{1}{2}\left(1-\frac{R}{R+\Delta R}+\frac{1}{2 \pi} \sin \frac{2 \pi R}{R+\Delta R}\right)^{-1},
$$

where $R$ is a radius of the hole and $\Delta R$ is an electron layer thickness. The value of $\Delta R$ was estimated as $0.166 \mathrm{~nm}$. Because for polymers $\tau_{3}$ is the most relevant quantity we will focus on its behaviour. For all the samples $o$-Ps intensity $I_{3}$ does not change significantly and is equal to ca. $23 \%$

It can be seen from Fig. 4 that for the shorter tiles, i.e., $10 \mathrm{~mm}$ and $100 \mathrm{~mm}$ long, $\tau_{3}$ has the same value within the experimental error range and it is equal to ca. $2.20 \mathrm{~ns}$ which corresponds to the average free volume radius equal to $0.30 \mathrm{~nm}$. It should be mentioned that the values reported in the literature for ABS are slightly lower, $c a .2 .14$ ns $[12,13]$. It is worth noticing that for the $200 \mathrm{~mm}$ long tiles even higher values of $\tau_{3}, c a .2 .24 \mathrm{~ns}$ corresponding to the average free volume radius $0.31 \mathrm{~nm}$ are obtained. It can be expected that the differences in positron lifetime values caused by higher length of raster would be small. The change of the volume fraction of the weld interface caused by adjustments of infill line distance parameter in the samples is limited, thus its contribution to the positron lifetime spectrum is small. Additionally, in polymers the free volume holes are not uniform in size and the obtained values of $\tau_{3}$ and subsequently values of the free volume radii are averaged over some distribution. It seems that the effect however small is observed for the samples with the longest raster. The effect of different infill distance for the measured samples is negligible.

The farther investigations require enhancement of the interface weld volume fraction by for example using smaller nozzle or/and increasing the sample length. To- gether with results from different mechanical test the PALS method may enrich knowledge about influence of microstructure on strength and durability of additive manufacturing elements.

\section{Conclusions}

In the paper, there were presented the results of the positron lifetime measurements of ABS specimens manufactured by FDM process. The specimens of unidirectional print configuration with different raster length and different overlapping of filament roads were chosen to highlight the presence of weld interface in the build. Results shows that the length of raster has some small influence on $o$-Ps lifetime value causing its increase from $2.20 \mathrm{~ns}$ to $2.24 \mathrm{~ns}$ for specimens with the longer raster. The differences are not observed when it comes to variations in the infill line distance parameter in the samples.

\section{Acknowledgments}

The authors wish to thank Ms. Aleksandra Gawron at 3D FLY S.C. for preparing the samples.

\section{References}

[1] B.N. Turner, R. Strong, S.A. Gold, Rapid Prototyping J. 20, 192 (2015).

[2] U.K. Roopavath, D.M. Kalaskar, in: 3D Printing in Medicine, Ed. D.M. Kalaskar, Woodhead Publ., 2017, p. 1.

[3] S. Curran, P. Chambon, R. Lind, L. Love, SAE Technical Paper, 2016-01-0328 (2016).

[4] F.P. Bos, R.J.M. Wolfs, Z.Y. Ahmed, T.A.M. Salet, Virt. Phys. Prototyp. 11, 209 (2016).

[5] R. Marissen, D. Schudy, A.V.J.M. Kemp, S.M.H. Coolen, W.G. Duijzings, A. Van Der Pol, A.J. Van Gulick, J. Mater. Sci. 36, 4167 (2001).

[6] D.P. Cole, J.C. Riddick, H.M. Iftekhar Jaim, K.E. Strawhecker, N.E. Zander, Appl. Polym. Sci. 133, 30 (2016).

[7] M.S. Hossain, J. Ramos, D. Espalin, M. Perez, R. Wicker, in: 24th Int. SFF Symp. An Additive Manufacturing Conf. SFF 2013, University of Texas at Austin, 2013, p. 380.

[8] L. Li, Q. Sun, C. Bellehumeur, P. Gu, J. Manuf. Process. 4, 129 (2002).

[9] J. Kansy, Nucl. Instrum. Methods A 374, 235 (1996).

[10] S.T. Tao, J. Chem. Phys. 56, 5499 (1972).

[11] N. Eldrup, D. Lightbody, J.N. Sherwood, Chem. Phys. 63, 51 (1981).

[12] G. Dlubek, M.A. Alam, M. Stolp, H.-J. Radusch, J. Polym. Sci. B Polym. Phys. 37, 1749 (1999).

[13] B.E. Tiganis, L.S. Burn, P. Davis, A.J. Hill, Polym. Degrad. Stabil. 76, 425 (2002). 